\title{
O FORMALISMO JURÍDICO DE ERNEST WEINRIB E SEUS REFLEXOS NA TEORIA DA RESPONSABILIDADE CIVIL
}

Tese apresentada à Faculdade de Direito da Universidade de São Paulo, na área de concentração de Filosofia e Teoria Geral do Direito, como requisito parcial para obtenção do título de doutora, sob a orientação do professor doutor Ronaldo Porto Macedo Junior.

FACULDADE DE DIREITO DA UNIVERSIDADE DE SÃO PAULO

SÃO PAULO

2012 


\section{AGRADECIMENTOS}

Ao meu orientador, professor Ronaldo Porto Macedo Junior, por esses sete anos de orientação atenta e interessada que foram absolutamente determinantes para o meu desenvolvimento intelectual e profissional.

Aos professores José Reinaldo de Lima Lopes e Marcos Paulo Veríssimo, membros dabanca de qualificação, cujas críticas e sugestões ao projeto foram fundamentais para a melhor estruturação desta tese.

Ao Fox International Fellowship Program, nas pessoas de Joseph e Alice Fox, pela bolsa de estudos que me permitiu permanecer na Yale University como pesquisadora visitante entre agosto de 2010 e junho de 2011. Esse programa cumpre integralmente com seu objetivo de propiciar uma experiência acadêmica e de vida absolutamente única. Aos meus queridos amigos do Fox Program e da Yale Law School, com quem dividi os melhores momentos do meu ano em New Haven. Auriane, Andrés, Carmit, Diego, Eduardo, James, Natasha, William, Thomaz e Tarsila (yalie honorária), sei que vocês são amigos para a vida toda.

À professora Mariana Mota Prado, por me ter aberto as portas da Toronto University Faculty of Law e atuado como minha tutora durante o período em que lá estive como pesquisadora visitante. Ao professor Ernest Weinrib, pela amabilidade com que me recebeu e abriu sua agenda para uma maratona de reuniões nas quatro semanas que lá passei. A disposição que demonstrou em discutir comigo suas ideias, os objetivos desta tese e muitos outros temas, como o futuro da educação e do ensino jurídico no Brasil e no Canadá, foi tanto valiosa quanto inspiradora. Embora curto, esse período em Toronto foi fundamental para que os capítulos em que trato do formalismo jurídico weinribiano ganhassem consistência e precisão. Ao Henrique, irmão querido, pela acolhida em Toronto. 
Aos meus pais, Nicolau e Carmen, por tudo. Ao Guilherme Paiva, pelo apoio no início desta caminhada e aos queridos Andrés Hincapié, Carmit Valensi, Carol Haber, Carol Ranzani, Cris Emy, Daniel Arbix, Daniela Gabbay, Evy Marques, Flavia Mange, James Cameron, Mariana Stelini, Melissa Mestriner e Pedro Abramovay, por terem me ajudado a concluí-la. Agradeço especialmente à Flavia, pela amizade, estímulo e companheirismo nesses últimos meses de redação da tese e ao James, pelas conversas nos finais de tarde. Sem vocês, eu simplesmente não teria chegado até aqui. 


\section{RESUMO}

Este trabalho trata da relação entre a teoria formalista do direito elaborada por Ernest Weinrib e sua teoria de fundamentação da responsabilidade civil derivada do formalismo. O objetivo do trabalho é mostrar que, apesar de o formalismo apresentar uma formulação distintiva e inovadora de racionalidade jurídica, que enfatiza a relação entre forma e conteúdo do direito, o modelo de racionalidade jurídica do formalismo não fornece uma explicação adequada para algumas áreas do direito e, especificamente no caso da responsabilidade civil, da responsabilidade objetiva, que, nessa visão, é considerada um erro jurídico.

O formalismo jurídico weinribiano é um projeto teórico ambicioso que a um só tempo procura rechaçar posições céticas - como os estudos críticos do direito (critical legal studies) — e posições instrumentalistas, especialmente a análise econômica do direito e o positivismo jurídico. Ele apresenta uma teoria geral que mostra o direito como um lócus de racionalidade moral imanente e um método, fundado na inteligibilidade imanente, para compreender essa racionalidade e ver o direito como um fenômeno autônomo em relação à política e a outras esferas de racionalidade.

O método formalista permite a intelecção do direito como um fenômeno imanentemente coerente por meio da apreensão das estruturas organizadoras e justificadoras implícitas nos arranjos jurídicos, isto é, as formas da justiça corretiva e da justiça distributiva. Com base nisso, o formalismo sustenta que a forma imanente ao direito privado e, consequentemente, à responsabilidade civil é a justiça corretiva.

Este trabalho reconstrói criticamente as principais teses que integram o formalismo e que são fundamentais para entender a rejeição à responsabilidade objetiva, considerada uma monstruosidade conceitual, com especial ênfase na premissa epistemológica formalista da inteligibilidade imanente como a melhor maneira de se compreender o direito e na premissa da coerência estrutural que os arranjos jurídicos devem apresentar.

A tese discute o modo como o formalismo jurídico se insere no debate metodológico contemporâneo, definindo o formalismo jurídico como uma teoria de avaliação e justificação jurídica e, portanto, dotada de uma dimensão prescritiva, e apresentando o impacto dessa definição na postura que o formalismo assume em relação à responsabilidade objetiva. 
A tese ainda analisa a relação entre essa dimensão prescritiva e o posicionamento do formalismo em relação à responsabilidade objetiva, rotulando-a de erro jurídico, e conclui com uma discussão sobre as razões desse posicionamento - que, argumentase, é equivocado - , que se baseiam na assunção das premissas da inteligibilidade imanente e do critério de coerência estrita que marcam o método de conhecimento formalista.

Palavras-chave: Ernest Weinrib. Formalismo. Instrumentalismo. Funcionalismo. Teoria prescritiva. Responsabilidade civil. Responsabilidade objetiva. Justiça corretiva. Justiça distributiva. 


\begin{abstract}
This dissertation focuses on the relationship between juridical formalism as elaborated by Ernest Weinrib and his theory of the foundation of tort law. The dissertation argues that despite formalism's distinctive and innovative account of legal rationality that emphasizes the relationship between law's form and substance, this account does not provide an adequate explanation for specific areas of law. Particularly in the case of tort law it does not adequately explain strict liability, which is deemed a juridical error.

Weinrib's juridical formalism is an ambitious theoretical project that challenges: skeptical accounts of law, such as critical legal studies; instrumentalists' account of law exemplified by economical analysis of law; and juridical positivism. Weinrib's theory attempts to explain law as a locus of immanent moral rationality. The theory is based on a methodology that adopts the premise of immanent intelligibility as a way to comprehend this rationality. This approach allows the theorist to grasp law as a phenomenon autonomous from politics and other spheres of rationality.

The formalist method allows the intelligibility of law as an immanently coherent phenomenon through the apprehension of the organizing and justifying structures implicit in juridical arrangements, i.e., the forms of corrective justice and distributive justice with which formalism argues that the immanent form of private law and, therefore, of tort law is corrective justice.

This dissertation reconstructs critically the main theses that support formalism, which are essential to understanding its rejection of strict liability - considered a conceptual monstrosity. This dissertation focuses on the formalist epistemological assumption of immanent intelligibility as the best way of comprehending law and on the premise of internal structural coherence that juridical arrangements should display.

This thesis discusses the way in which juridical formalism can be located within the contemporary methodological debate, and defines formalism as an evaluative and justificatory juridical theory. As such, formalism encompasses a prescriptive dimension. This dissertation also identifies how the definition of formalism as a prescriptive theory impacts on its analysis of strict liability
\end{abstract}


The dissertation then analyses the relationship between this prescriptive dimension and the formalist position regarding strict liability. It concludes that formalism's mistake regarding strict liability can be traced back to its epistemological assumptions regarding immanent intelligibility and coherence.

Key-words: Ernest Weinrib. Formalism. Instrumentalism. Functionalism. Prescriptive theory. Tort law. Strict liability. Corrective justice. Distributive justice. 


\section{SUMÁRIO}

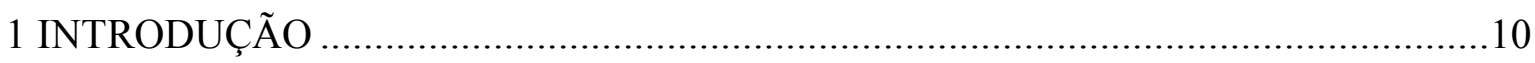

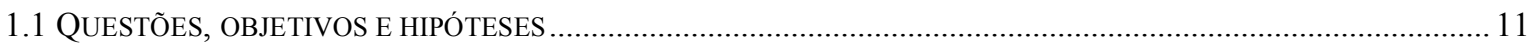

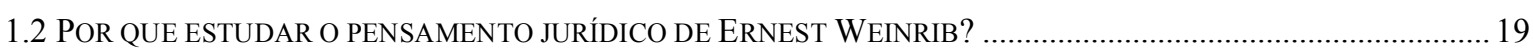

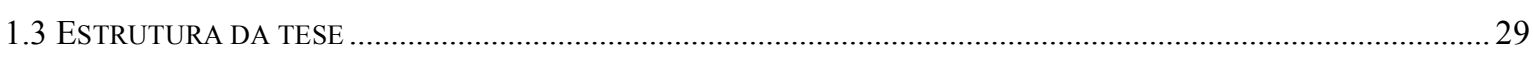

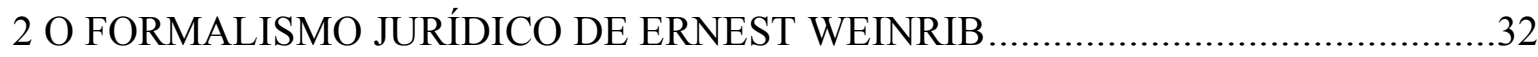

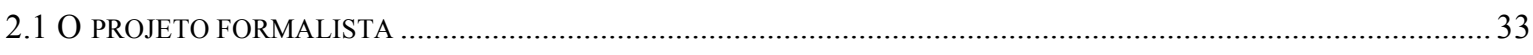

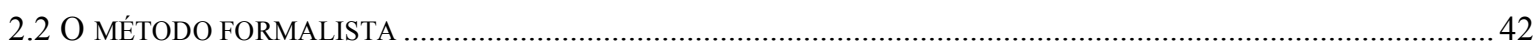

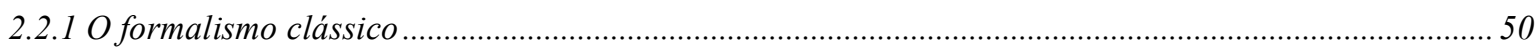

2.2.2 A rejeição às concepções instrumentalistas e funcionalistas do direito ................................................. 52

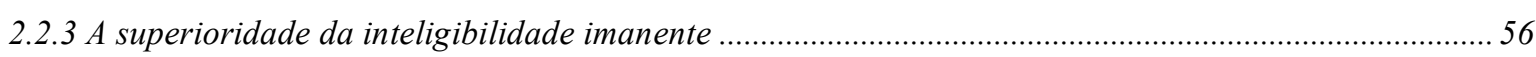

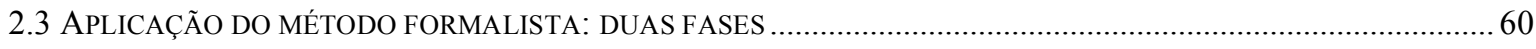

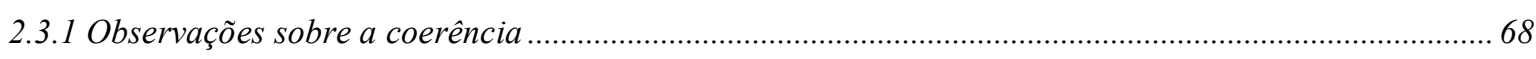

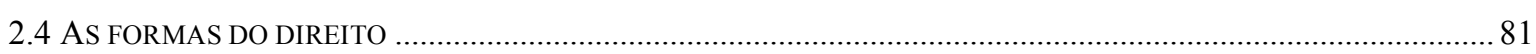

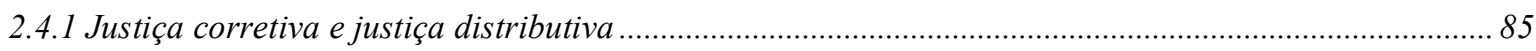

2.4.2 As teses da irredutibilidade e da incomunicabilidade das formas ............................................................. 93

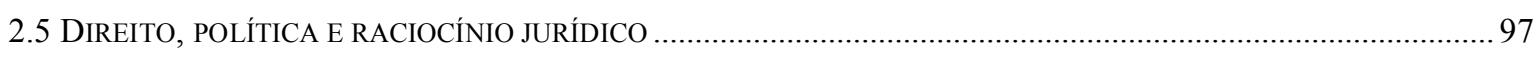

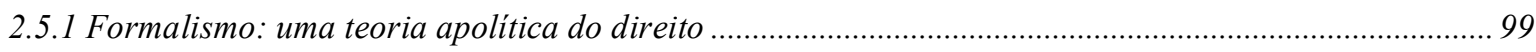

2.5.2 Papel dos tribunais e raciocínio jurídico (legal reasoning)................................................................. 101

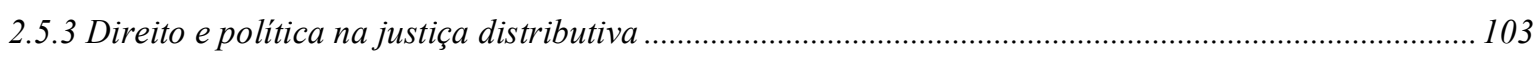

2.5.4 A ausência de relação entre política e justiça corretiva.......................................................................... 110

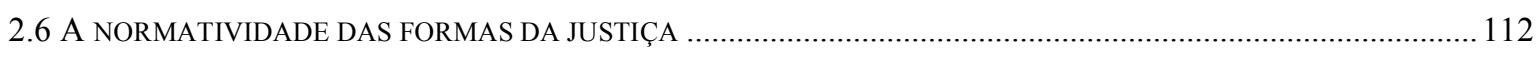

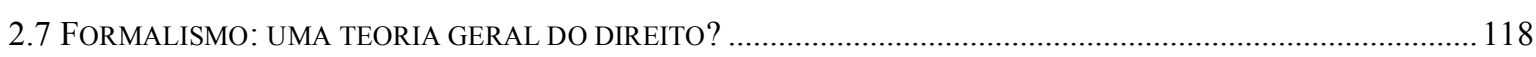

\section{OBJETIVOS DA TEORIA DO DIREITO: UMA DISCUSSÃO}

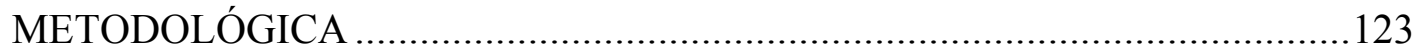

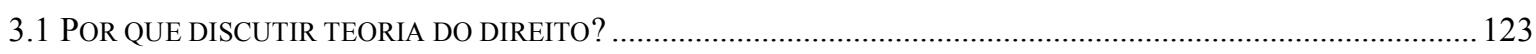

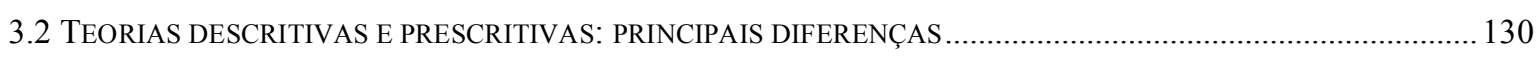

3.3 JOHN FINNIS E A DEFESA DE UMA TEORIA PRESCRITIVA (NORMATIVA) DO DIREITO ……………………….... 139

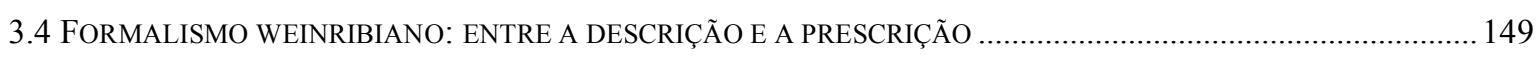

3.5 UM OUTRO OLHAR SOBRE A DISCUSSÃO DE METODOLOGIA EM TEORIA DO DIREITO ...................................... 153

3.5.1 Uma sugestão de superação da dicotomia descrição/prescrição ......................................................... 156

3.5.2 John Finnis e as teses da avaliação moral e da justificação moral do direito ....................................... 164

3.5.3 Joseph Raz e a tese da avaliação indireta do direito............................................................................ 170

3.6 O LUGAR DO FORMALISMO WEINRIBIANO NO DEBATE METODOLÓGICO......................................................... 174 
4 A RELAÇÃO ENTRE A TEORIA FORMALISTA DO DIREITO E A FUNDAMENTAÇÃO DA RESPONSABILIDADE CIVIL

4.1 O FORMALISMO E A FUNDAMENTAÇÃO DA RESPONSABILIDADE CIVIL ................................................... 183

4.2 RESPONSABILIDADE BASEADA NA CULPA (NEGLIGENCE LAW): O PERFEITO ENCAIXE TEÓRICO ..............194

4.3 A RESPONSABILIDADE OBJETIVA: MONSTRUOSIDADE CONCEITUAL? .......................................................... 197

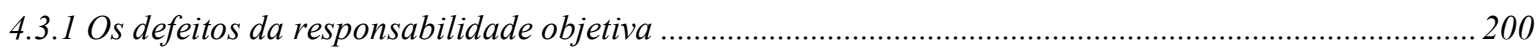

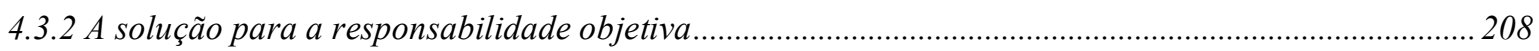

5 PROBLEMAS DO FORMALISMO JURÍDICO: PADRÃO DE INTELIGIBILIDADE E CRITÉRIO DE COERÊNCIA....................................217

5.1 O PARADIGMA DA INTELIGIBILIDADE INTERNA E A VISÃO ESSENCIALISTA DO DIREITO ..............................221

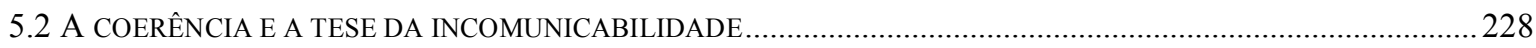

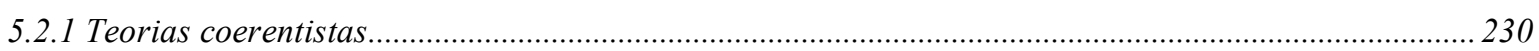

5.2.2 Formalismo e coerência: uma segunda análise ........................................................................... 235

5.2.3 Irredutibilidade e incomunicabilidade entre justiça corretiva e justiça distributiva .........................241

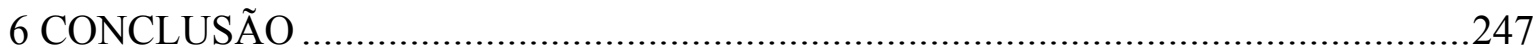

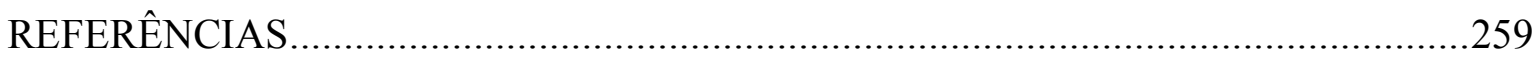

